\title{
IncRNA DLG1-AS1 Promotes Cell Proliferation by Competitively Binding with miR-107 and Up-Regulating ZHX1 Expression in Cervical Cancer
}

\author{
Xiaohui Rui Yun Xu Yaqing Huang Linjuan Ji Xiping Jiang \\ Department of Gynecology, The Third Affiliated Hospital of Soochow University, Changzhou, China
}

\section{Key Words}

DLG1-AS1 • miR-107 • ZHX1 • Cervical cancer

\begin{abstract}
Background/Aims: Recent studies have revealed that long non-coding RNAs (IncRNAs) are involved in the occurrence and development of various tumors, thereby attracting increasing attention from researchers. The important biological functions of IncRNAs have been recognized gradually, but their mechanism in cervical cancer remains unclear. Methods: Differentially expressed IncRNAs in cervical cancer and para-carcinoma tissues were identified by screening using an IncRNA array, and candidate IncRNAs were verified by quantitative real-time PCR. A series of bioinformatics and molecular biological methods were adopted to investigate the interactions among IncRNAs, microRNAs (miRNAs), and miRNA target genes in cervical cancer. Cell viability was measured using a Cell Counting Kit-8 assay. Results: DLG1AS1 was the most significantly up-regulated IncRNA in cervical cancer tissues, and it was confirmed that cervical cancer patients with high DLG1-AS1 expression had a poor prognosis. Down-regulation of DLG1-AS1 expression suppressed the proliferation of cervical cancer cells. Further investigation revealed that DLG1-AS1 eliminated the inhibition of miR-107 on the expression of its target gene ZHX1 by competitively binding to miR-107. Moreover, rescue assays proved that the effect of DLG1-AS1 on the proliferation of cervical cancer cells was dependent on miR-107. Conclusion: DLG1-AS1/miR-107/ZHX1 can form a competitive endogenous RNA network that regulates the proliferation of cervical cancer cells, resulting in tumor progression.




\section{Cellular Physiology Cell Physiol Biochem 2018;49:1792-1803 \begin{tabular}{ll|l} 
and Biochemistry Published online: 20 September, 2018 & $\begin{array}{l}\text { (c) } 2018 \text { The Author(s). Published by S. Karger AG, Basel } \\
\text { www.karger.com/cpb }\end{array}$ \\
\hline
\end{tabular} \\ Rui et al.: Effect of IncRNA DLG1-AS1 in Cervical Cancer}

\section{Introduction}

Cervical cancer is a malignant tumor with a morbidity rate second only to that of breast cancer. Approximately 470, 000 new cases and 233, 000 deaths occur each year globally, approximately $90 \%$ of which occur in developing countries, showing a younger trend [1]. There are approximately 150, 000 new cases each year in China [2], which poses a serious threat to the health of women. With the popularization of cervical cancer screening and the timely treatment of precancerous lesions of the uterine cervix, the incidence rate of cervical cancer has decreased profoundly in developed regions. However, its incidence remains at a higher level in undeveloped regions due to the lack of or irregular implementation of screening. In recent years, despite great progress in treatment methods and techniques, the prognosis of patients with mid-late cervical cancer is still unsatisfactory. Therefore, it is particularly urgent to identify molecular markers for the early screening of cervical cancer as well as new specific therapeutic targets.

Studies have shown that the abnormal expression of microRNAs (miRNAs) plays an important role in the occurrence, progression, diagnosis, treatment, and prognosis of most tumors, including cervical cancer $[3,4]$. miRNAs are a type of small non-coding singlestranded RNA that can bind to the $3^{\prime}$-untranslated region (UTR) of target gene mRNA via complementary base pairing, inhibiting the degradation or translation of target gene mRNA, regulating gene expression at the post-transcriptional level, and participating in the regulation of cellular processes, such as inflammatory and stress responses, differentiation, apoptosis, and migration [5-7]. The abnormal expression of miRNAs in tumors is a research hotspot in the field of oncology. A variety of miRNAs that regulate the biological behavior of cervical cells through different target genes and participate in the occurrence, invasion, and metastasis of cervical cancer have been observed to be expressed abnormally in cervical cancer [8-10].

Long non-coding RNAs (lncRNAs) are novel non-coding RNAs more than 200 nucleotides in length. Compared with miRNAs, IncRNAs have complex structures and diversified expression regulation. Studies have implicated lncRNAs in important roles, such as protein function regulation, genomic imprinting, transcription, and post-transcriptional regulation [11]. Recent studies have shown that IncRNAs can function as competitive endogenous RNAs (ceRNAs) and bind to miRNA recognition elements in the 3 '-UTR of mRNAs to release the inhibition or degradation of miRNAs against target genes, thereby regulating the mRNA and protein expression of target genes [12-15]. The important biological functions of lncRNAs have been recognized gradually, but their mechanism in cervical cancer remains unclear.

In this study, differentially expressed IncRNAs were screened for in cervical cancer and para-carcinoma tissues by using an IncRNA chip, and the screening results were verified in the samples via quantitative real-time PCR (qRT-PCR). DLG1-AS1 (ENSG00000227375), the IncRNA with the most significant differential expression, was selected to explore the pathway and regulation mechanism of the DLG1-AS1/miRNA/target gene axis and to examine its effect on the proliferation of cervical cancer cells, providing new molecular targets for the diagnosis and treatment of cervical cancer.

\section{Materials and Methods}

Tissue specimens

A total of 112 patients with cervical cancer receiving operative treatment at the Third Affiliated Hospital of Soochow University from 2009 to 2014 were selected in the present study. The collected tissue specimens were stored in liquid nitrogen at $-80^{\circ} \mathrm{C}$. The collection of clinical specimens was approved by the Ethics Committee of the Third Affiliated Hospital of Soochow University and performed in accordance with the Declaration of Helsinki. All patients provided informed consent. 


\section{Cellular Physiology Cell Physiol Biochem 2018;49:1792-1803 \begin{tabular}{ll|l} 
and Biochemistry Published online: 20 September, 2018 & $\begin{array}{l}\text { (c) } 2018 \text { The Author(s). Published by S. Karger AG, Basel } \\
\text { www.karger.com/cpb }\end{array}$ \\
\hline
\end{tabular}}

Rui et al.: Effect of IncRNA DLG1-AS1 in Cervical Cancer

\section{LncRNA chip detection}

The expression of IncRNAs in cervical cancer and para-carcinoma tissues was analyzed by using an Arraystar Human LncRNA Array v3.0 (Arraystar, Inc., Rockville, MD) according to the manufacturer's instructions. The measured data were analyzed statistically, and lncRNAs with significant differential expression were screened in the two groups of chips (screening criteria: fold change $>3, P<0.01$ in t-test).

\section{Cell culture and transfection}

The normal cervical epithelial cell line End1/E6E7 and the cervical cancer cell lines HeLa, SiHa, Caski, and C4-1 were purchased from the American Type Culture Collection (Manassas, VA). The cells were incubated in Dulbecco's modified Eagle's medium containing 1\% penicillin/streptomycin and 10\% fetal bovine serum in an incubator with $5 \% \mathrm{CO}_{2}$ at $37^{\circ} \mathrm{C}$, followed by passaging once every 2-3 days until the logarithmic growth phase.

The DLG1-AS1-overexpression plasmid, DLG1-AS1 small interfering RNAs (siRNAs), and negative control were purchased from Sigma-Aldrich (St. Louis, MO). The miR-107 mimic, miR-107 inhibitor, and negative control were purchased from RiboBio (Guangzhou, China). The cells were transfected by using Lipofectamine 2000 as the carrier, according to the instructions of the kit. Transfection efficiency was observed under a fluorescence microscope at $24 \mathrm{~h}$ after transfection to determine whether subsequent experiments could be performed. Then, the cells were collected at the appropriate time points according to the experimental requirements of the further experiments.

qRT-PCR

Fresh biopsy specimens were collected and frozen at $-80^{\circ} \mathrm{C}$ and subsequently ground to powder by using liquid nitrogen. Total RNA was extracted by using the TRIzol reagent and purified to remove genomic DNA, to eliminate its interference on miRNAs and lncRNAs. The concentration and purity of RNA were measured with a nucleic acid protein tester. Primer design and reverse transcription into cDNA and qRTPCR were performed according to the instructions of the Takara PrimeScript ${ }^{\mathrm{TM}}$ RT Master Mix Kit (Takara Bio Inc., Kusatsu, Japan). Primer information is shown in Table 1.

\section{Prediction and validation of DLG1-AS1 combined with miR-107}

miRNAs that could bind to IncRNA DLG1-AS1 via complementary base pairing were predicted jointly by using miRcode (http://www.mircode.org/), starBase (http://starbase.sysu.edu.cn/index.php), and RegRNA (http://regrna2.mbc.nctu.edu.tw/index.html). A dual-luciferase reporter assay was performed to verify the ability of DLG1-AS1 to bind to the candidate miRNA. The wild-type and mutant-type fluorescence plasmids psiCheck2-DLG1-AS1-Luc and psiCheck2-DLG1-AS1-MUT-Luc, respectively, were constructed. HeLa cells were co-transfected with the miR-107 mimic, psiCheck2-DLG1-AS1-Luc, and psiCheck2-DLG1AS1-MUT-Luc vector. Luciferase activity was detected in the experimental and control groups by using a dual-luciferase reporter assay.

\section{Prediction and validation of miR-107 target genes}

The target genes to which miR-107 can bind directly were predicted jointly through TargetScan (http:// www.targetscan.org/vert_71/), miRDB (http://www.mirdb.org/), and RNA22 (https://cm.jefferson.edu/ rna22/). The wild-type and mutant-type fluorescence plasmids psiCheck2- ZHX1-Luc and psiCheck2ZHX1-MUT-Luc, respectively, were constructed. ZHX1 was cotransfected with the miR-205 mimic, psiCheck2- ZHX1-Luc, and psiCheck2- ZHX1-MUT-Luc vectors. Luciferase activity was detected in the experimental and control groups by using a dualluciferase reporter assay.

Table 1. Primer sequences used for qRT-PCR assays

\begin{tabular}{lr}
\hline Gene & Sequence of the primers \\
\hline DLG1-AS1-Forward & 5'-CCGAAACTTTCCGCCAAGATG-3' \\
DLG1-AS1-Reverse & 5'-CCTCACTTCCCATTGGCTGAG-3' \\
ZHX1-Forward & 5'-GCAGGCGAAAATCAACAACAC-3' \\
ZHX1-Reverse & 5'-TAAGCACAGGAGGACCTTCAT-3' \\
GAPDH-Forward & 5'-CTCACCGGATGCACCAATGTT-3' \\
GAPDH-Reverse & 5'-CGCGTTGCTCACAATGTTCAT-3' \\
miR-107 & 5'-AGCAGCAUUGUACAGGGCUAUCA-3' \\
U6 & 5'-CGCAAGGATGACACGCAAATTC-3' \\
\hline
\end{tabular}




\section{Cellular Physiology Cell Physiol Biochem 2018;49:1792-1803

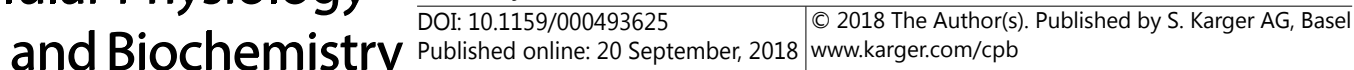

\section{Detection of cell proliferation activity via Cell Counting Kit-8 assay}

The proliferation of cervical cancer cells was detected by using a Cell Counting Kit-8 (CCK-8) assay (Dojindo Molecular Technologies, Kumamoto, Japan). Cells in the logarithmic growth phase in each group were collected and inoculated into a 96 -well plate at a density of $1.0 \times 10^{4}$ cells $/ \mathrm{mL}$. Then, $100 \mu \mathrm{L}$ of the cell suspension was added to each well, and 3 repeated wells were set in each group. Cell growth was observed. At different time points, $10 \mu \mathrm{L}$ CCK-8 solution was added to each well, followed by incubation at $37^{\circ} \mathrm{C}$ for another $4 \mathrm{~h}$. After $4 \mathrm{~h}$, the culture was terminated, and the absorbance of each well at a wavelength of 450 $\mathrm{nm}$ (A450) was measured by using a microplate reader to reflect the number of living cells indirectly. The cell proliferation rate (\%) was calculated as: (mean $\mathrm{A} 450_{\text {experimental well }}$ - mean $\left.\mathrm{A} 450_{\text {blank well }}\right) /\left(\right.$ mean $\mathrm{A} 450_{\text {control }}$ well - mean $\left.\mathrm{A}_{450_{\text {blank well }}}\right) \times 100$. The experiment was repeated 3 times.

\section{Statistical methods}

Student's t-test and one-way analysis of variance were used to analyze the differences between two groups and among the groups. Spearman's correlation test was performed to analyze the correlations among variables. SPSS 20.0 (SPSS Inc., Chicago, IL) and GraphPad Prism 5 (Graphpad Software, Inc., La Jolla, CA) software were used for statistical and cartographic analyses. The results are presented as the mean \pm standard error. The inspection level was $\alpha=0.05$ on both sides. The significance levels included $P<0.05, P$ $<0.01$, and $P<0.001$.

\section{Results}

Screening of differentially expressed IncRNAs in cervical cancer

LncRNA chip analysis was performed for 10 pairs of cervical cancer and para-carcinoma tissues to identify differentially expressed lncRNAs. A differential expression fold change $>3$ and $P<0.01$ in the t-test in cervical cancer and para-carcinoma tissues were used as the selection criteria; a total of 82 lncRNAs were up-regulated and 40 lncRNAs were downregulated in cervical cancer tissues (Supplementary Fig. S1A - For all supplementary material see www.karger.com/10.1159/000493625/). We selected the 10 most significantly up-regulated lncRNAs in cervical cancer, namely, ENSG00000227375, ENSG00000251577, ENSG00000237352, ENSG00000239677, ENSG00000231768, ENSG00000253302, ENSG00000254510, ENSG00000235151, ENSG00000246985, and ENSG00000259275 (Supplementary Fig. S1B), and the 10 most significantly down-regulated lncRNAs in cervical cancer, namely, ENSG00000226286, ENSG00000248859, ENSG00000241180, ENSG00000223392, ENSG00000214894, ENSG00000254842, ENSG00000223685, ENSG00000260179, ENSG00000237517, and ENSG00000234928 (Supplementary Fig. S1C), followed by verification via qRT-PCR in 30 pairs of cervical cancer and para-carcinoma tissues. The results showed that ENSG00000227375 (DLG1-AS1) was the most significantly highly expressed IncRNA in cervical cancer. Thus, DLG1-AS1 was selected for further experiments.

DLG1-AS1 expression in cervical cancer and its correlation with the prognosis of patients

qRT-PCR was performed to detect the expression of DLG1-AS1 in cervical cancer and para-carcinoma tissues in 112 patients. The results showed that DLG1-AS1 was expressed at a significantly higher level in cervical cancer tissues than in para-carcinoma tissues $(P<$ 0.001 ) (Fig. 1A). The median expression value was selected as the cut-off value to differentiate between high/low expression. The overall survival (OS) of cervical cancer patients with high DLG1-AS1 expression was significantly inferior to that of patients with low DLG1-AS1 expression $(P=0.006)$ (Fig. 1B).

DLG1-AS1 expression in cervical cancer cells and its effect on cell proliferation

We examined the differences in DLG1-AS1 expression between the normal cervical epithelial cell line End1/E6E7 and the cervical cancer cell lines HeLa, SiHa, Caski, and C4-1, 


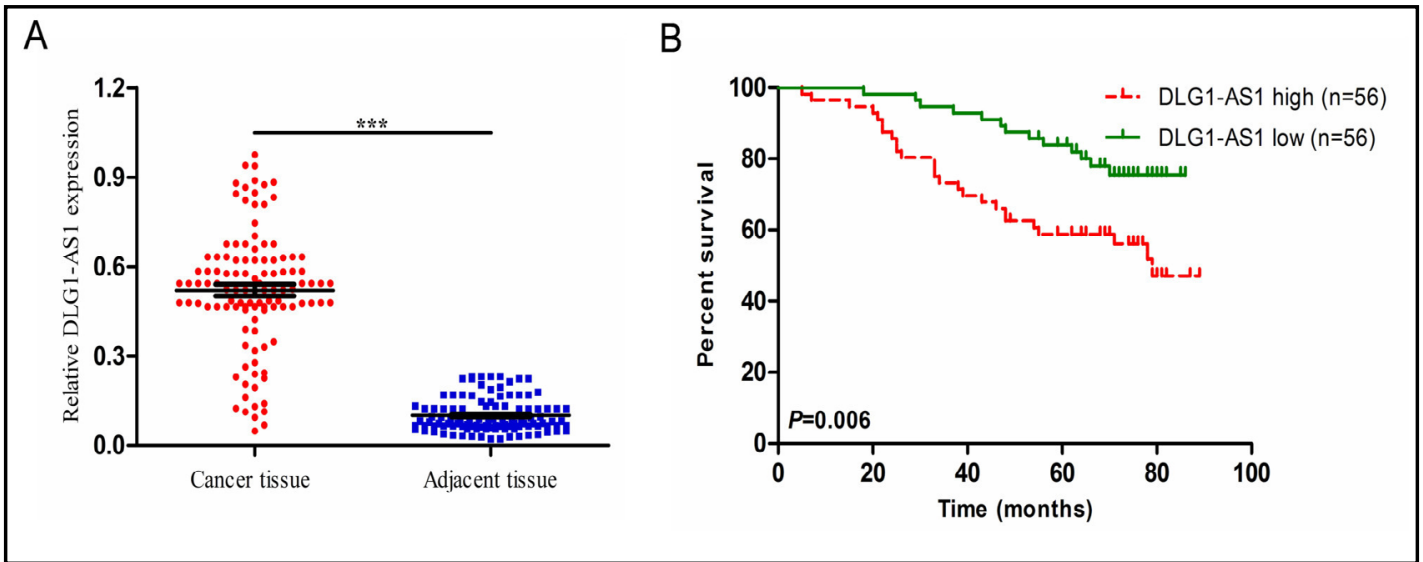

Fig. 1. DLG1-AS1 expression in cervical cancer and its correlation with prognosis of patients. A. The expression level of DLG1-AS1 in cervical cancer tissues is significantly higher than that in para-carcinoma tissue. B. The expression level of DLG1-AS1 is negatively correlated with the prognosis of patients with cervical cancer. OS of patients with high expression of DLG1-AS1 is significantly inferior to that of patients with low expression of DLG1-AS1 $(\mathrm{P}=0.006)$.

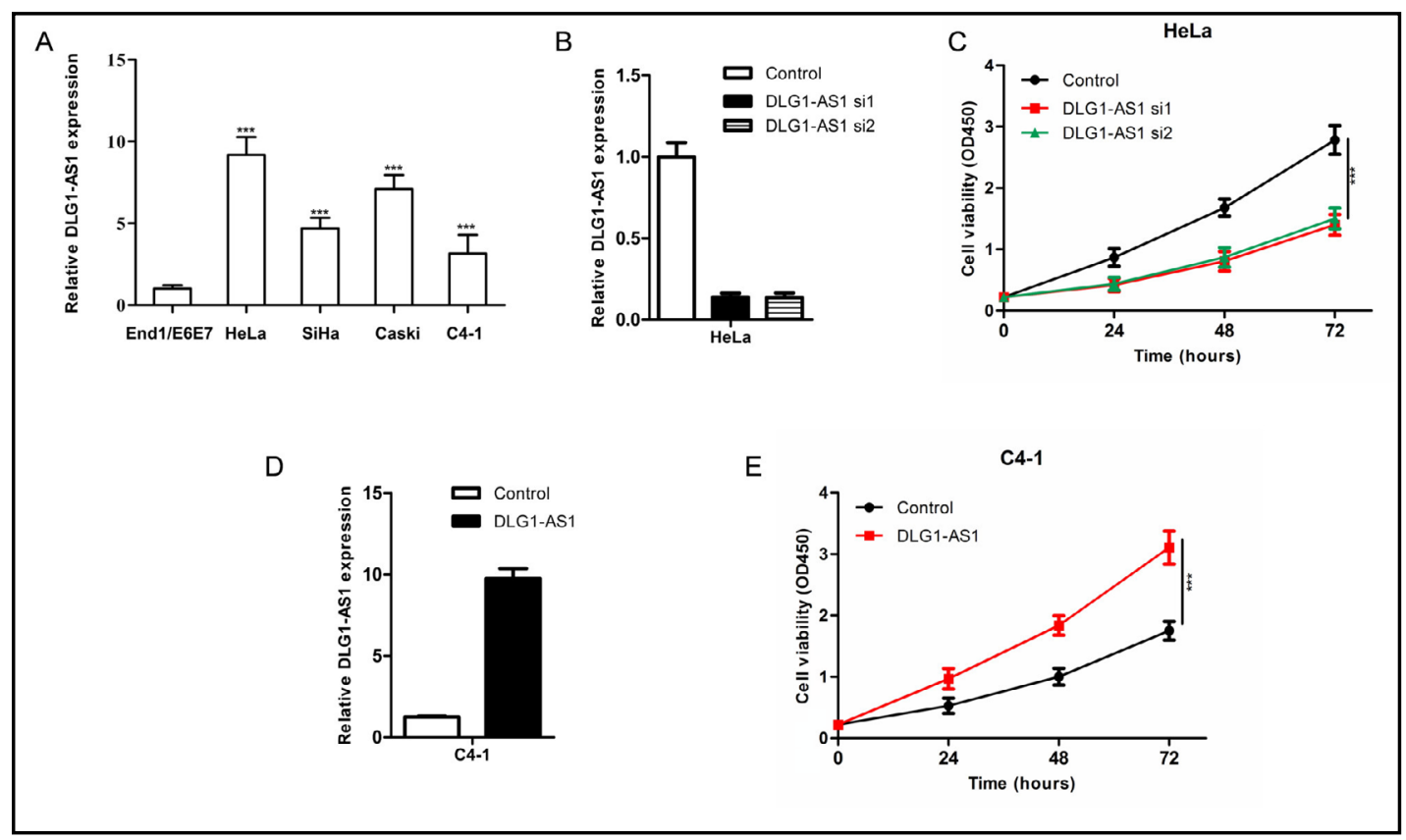

Fig. 2. Expression of DLG1-AS1 in cervical cancer cells and its effect on cell proliferation. A. Expression of DLG1-AS1 in normal cervical epithelial cell lines and x cervical cancer cell lines. The expression of DLG1-AS1 in cervical cancer cell lines (Hela, SiHa, Caski and C4-1) is significantly higher than that in cervical epithelial cell line End1/E6E7. B. After down-regulation of DLG1-AS1 expression in HeLa cell lines, interference efficiency is verified via qRT-PCR. C. After down-regulation of DLG1-AS1 expression, the proliferation of Hela cells is significantly decreased. D. After up-regulation of DLG1-AS1 expression in C4-1 cell line, interference efficiency is verified via qRT-PCR. E. After up-regulation of DLG1-AS1 expression, the proliferation of C4-1 cells is significantly enhanced.

and its expression was significantly higher in the cervical cancer cell lines than in the normal cervical epithelial cell line $(P<0.001$, Fig. 2A). DLG1-AS1 expression was highest in the HeLa cell line and lowest in the C4-1 cell line (Fig. 2A). 


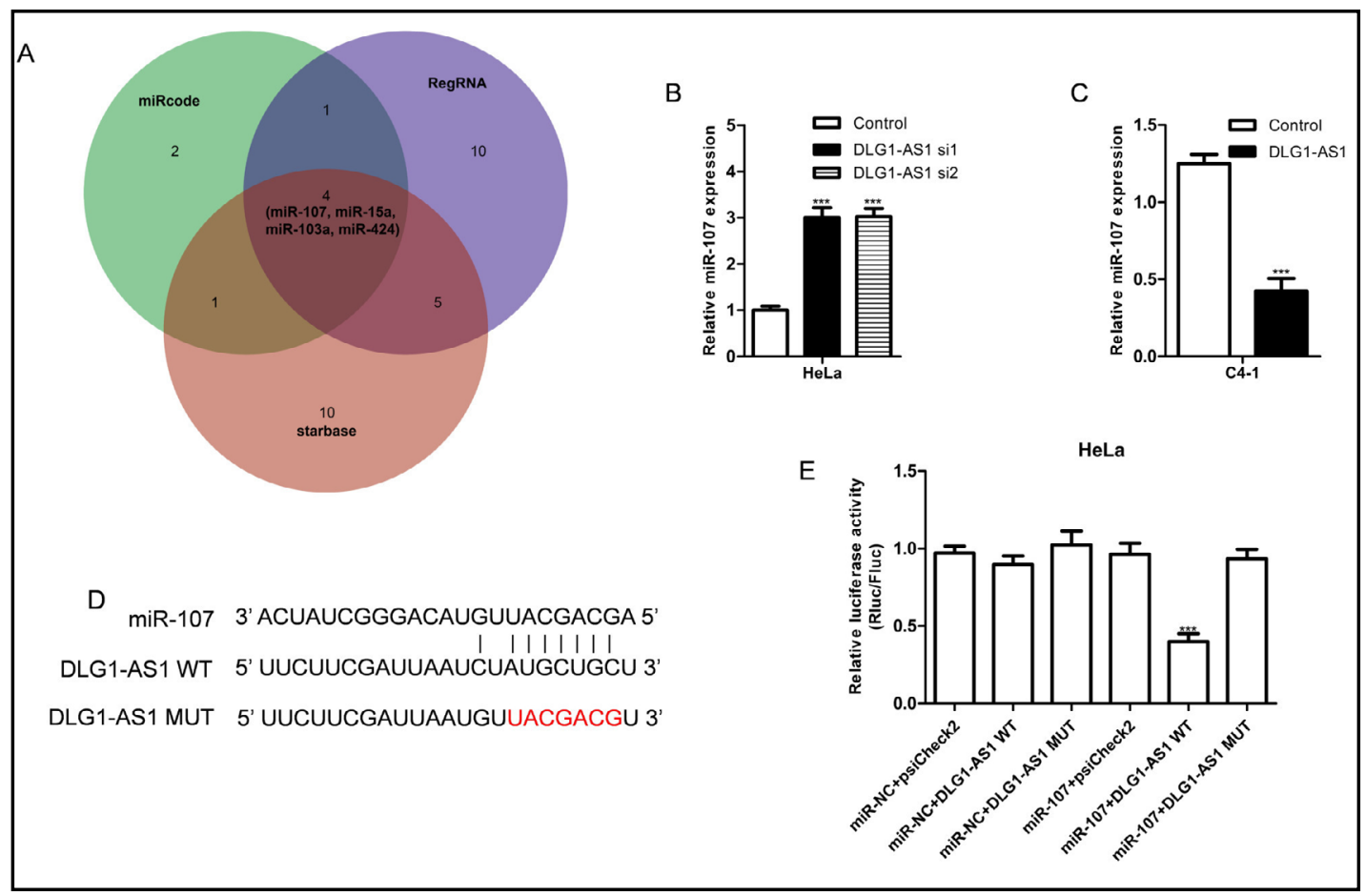

Fig. 3. Regulation of screening and verification of miRNAs via DLG1-AS1. A. miRcode, starBase and RegRNA are used to predict miRNAs that can bind to DLG1-AS1: miR-107, miR-15a, miR-103a and miR-424. B. After down-regulation of DLG1-AS1 expression in Hela cell lines, the miR-107 level is significantly elevated. C. After up-regulation of DLG1-AS1 expression in C4-1 cell lines, the miR-107 level is significantly reduced. D. miR-107 and DLG1-AS1 binding sequences and DLG1-AS1 mutation sequences. E. Luciferase reporter assay. Overexpression of miR-107 can significantly reduce the luciferase activity containing the wild-type DLGl-AS1 vector without reducing the luciferase activity containing the mutant-type vector or empty vector.

After the HeLa cell line was transfected with siRNA (si1 and si2) against DLG1-AS1, the expression of DLG1-AS1 was down-regulated, and the interference efficiency was verified via qRT-PCR $(P<0.001$, Fig. 2B). After DLG1-AS1 expression was down-regulated, cell proliferation was significantly decreased ( $P<0.001$, Fig. 2C).

After the C4-1 cell line was transfected with the pcDNA3.1-DLG1-AS1-overexpressing vector, the expression of DLG1-AS1 was up-regulated. Overexpression efficiency was verified via qRT-PCR $(P<0.001$, Fig. 2D). After DLG1-AS1 expression was up-regulated, cell proliferation was significantly enhanced $(P<0.001$, Fig. 2E).

\section{Screening and verification of miRNAs that can bind to DLG1-AS1}

MiRNAs that can bind to DLG1-AS1 via complementary base pairing were predicted by using the three databases miRcode, starBase, and RegRNA (Fig. 3A). A total of 4 miRNAs (miR-107, miR-15a, miR-103a, and miR-424) were predicted by the databases to bind to DLG1-AS1. After the HeLa cell line was transfected with siRNA (si1 and si2) against DLG1AS1, the expression of DLG1-AS1 was down-regulated, the level of miR-107 was significantly increased $(P<0.001$, Fig. 3B), and the levels of the other miRNAs remained unchanged. After the C4-1 cell line was transfected with the pcDNA3.1-DLG1-AS1-overexpression vector, DLG1-AS1 expression was up-regulated, the level of miR-107 was significantly decreased $(P$ $<0.001$, Fig. 3C), and the levels of the other miRNAs remained unchanged. Thus, miR-107 may be and miRNA regulated by DLG1-AS1.

After wild-type and mutant-type DLG1-AS1 sequences were cloned into the luciferase reporter plasmid psiCheck2 and co-transfected with the miR-107 mimic into HeLa cells, a luciferase reporter assay was performed (Fig. 3D). The results revealed that miR-107 


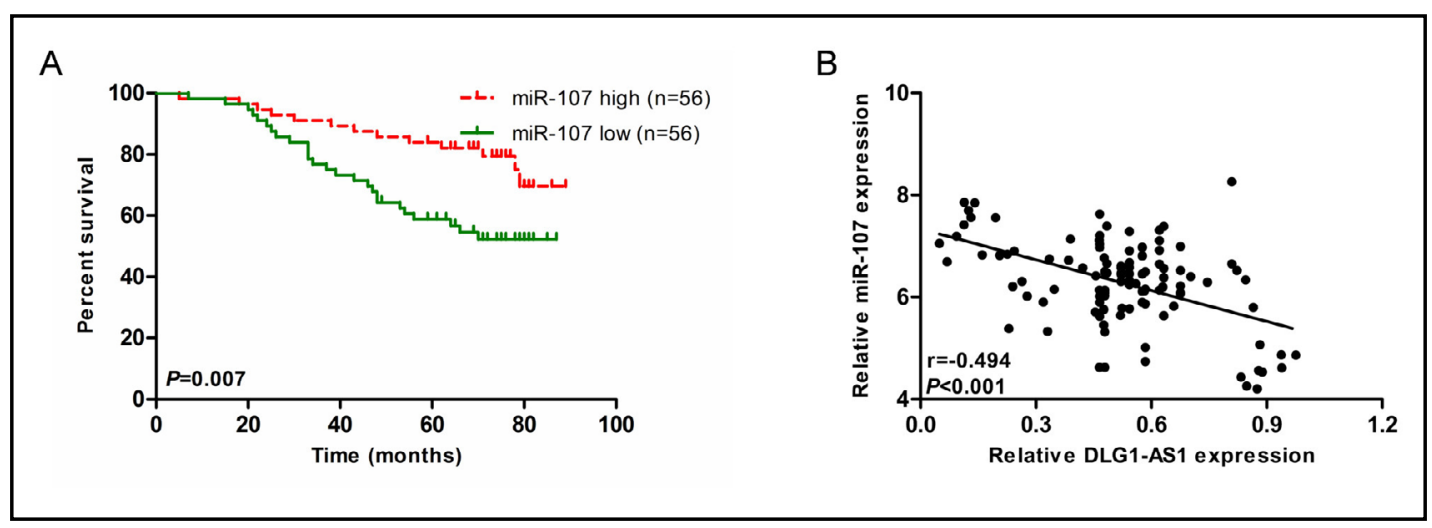

Fig. 4. Correlations of miR-107 expression with prognosis of patients and DLG1-AS1. A. Correlation between miR-107 expression and prognosis of patients with cervical cancer. OS of patients with high expression of miR-107 is significantly superior to that of patients with low expression of miR-107 ( $\mathrm{P}=0.007)$. B. Correlation between miR-107 expression and DLG1-AS1 in cervical cancer tissues. The expression level of miR-107 is negatively correlated with DLG1-AS1 ( $r=-0.494, \mathrm{P}<0.001)$.

overexpression significantly reduced the luciferase activity of the wild-type DLG1-AS1 vector $(P<0.001)$ without reducing that of the mutant-type vector or empty vector (Fig. $3 \mathrm{E}$ ), confirming that miR-107 can bind directly to DLG1-AS1 and that the mutated site is the location of this interaction.

Correlations of miR-107 expression with the prognosis of patients and DLG1-AS1

qRT-PCR was performed to detect the expression of miR-107 in 112 patients with cervical cancer, and the correlation between miR-107 expression and patient prognosis was analyzed. The results demonstrated that miR-107 expression was positively correlated with the OS of cervical cancer patients. The OS of patients with high miR-107 expression was significantly superior to that of patients with low miR-107 expression $(P=0.007)$ (Fig. 4A). In addition, Spearman's correlation test showed that the expression of miR-107 in 112 cases of cervical cancer was negatively correlated with that of DLG1-AS1 $(r=-0.494, P<0.001$, Fig. 4B).

\section{Screening and validation of miR-107 target genes}

A total of 7 miR-107 target genes were predicted by using TargetScan, miRDB, and RNA22: ANO3, ARMC1, ZHX1, AXIN2, MED26, RNF38, and TMEM47 (Fig. 5A). After miR-107 expression was up-regulated in the HeLa and C4-1 cell lines, the expression of ZHX1 was significantly decreased $(P<0.001$, Fig. 5B). After miR-107 expression was down-regulated in the HeLa and C4-1 cell lines, the expression level of ZHX1 was significantly increased $(P$ $<0.001$, Fig. 5C). There was no significant change in the expression of the other candidate target genes, suggesting that ZHX1 may be a target gene of miR-107. ZHX1 3'-UTR wildtype and mutant-type sequences were cloned into the luciferase reporter plasmid psiCheck2 and co-transfected with the miR-107 mimic into HeLa cells (Fig. 5D). The results showed that fluorescence intensity was significantly reduced following transfection with the wildtype ZHX1 fluorescence plasmid compared to transfection with the mutant-type ZHX1 fluorescence plasmid $(P<0.001$, Fig. 5E), confirming that ZHX1 can bind directly to miR-107 and that the mutation site is the location of this interaction.

\section{DLG1-AS1 regulates ZHX1 expression through competitive binding to miR-107}

As described previously, miR-107 expression was negatively correlated with DLG1-AS1 expression ( $\mathrm{r}=-0.494, P<0.001$, Fig. 4B). After DLG1-AS1 expression was down-regulated in the HeLa cell line, miR-107 expression was significantly increased $(P<0.001$, Fig. 4B), and miR-107 expression was significantly decreased after DLG1-AS1 expression in the 


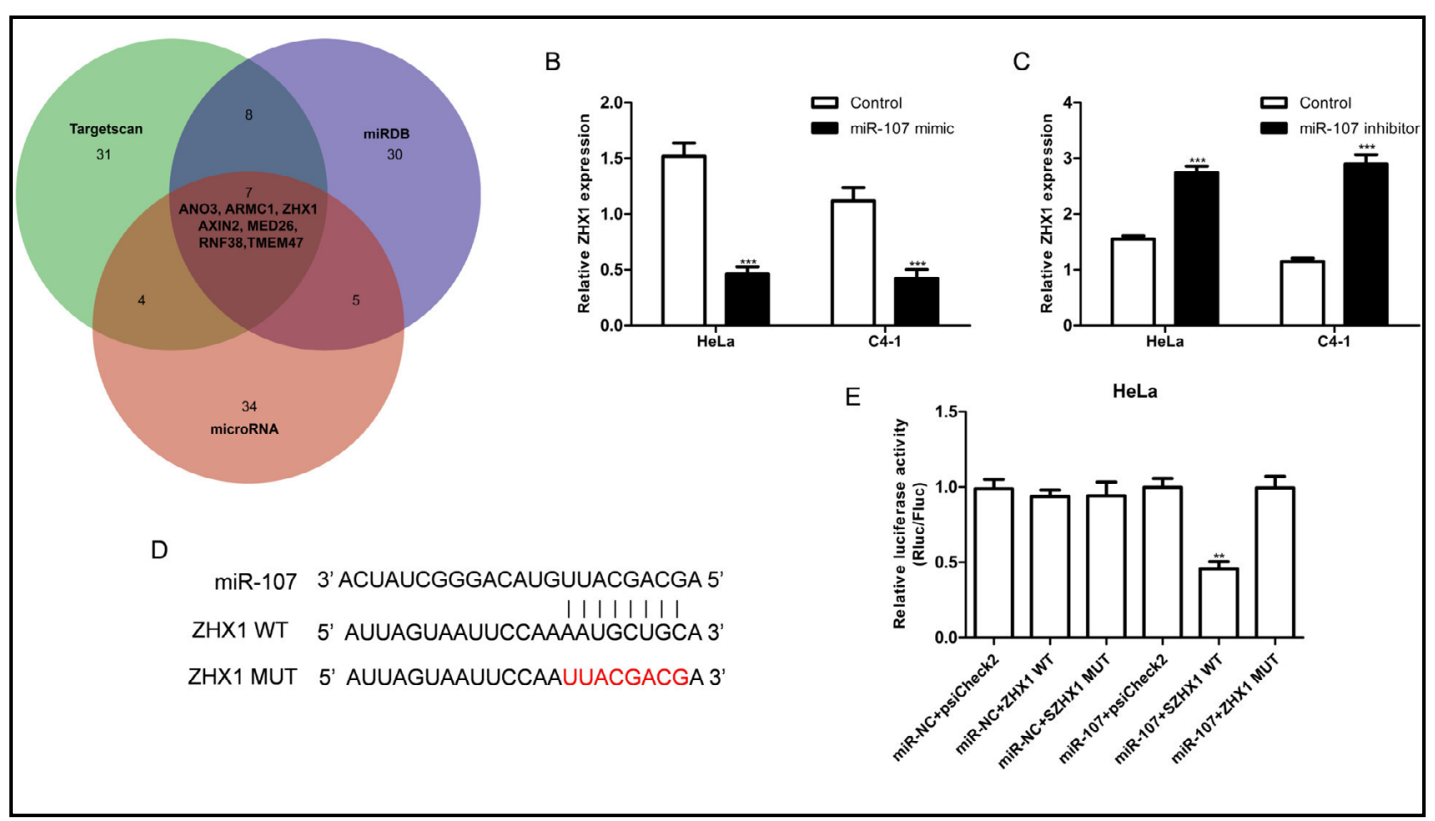

Fig. 5. Screening and validation of miR-107 target genes. A. Targetscan, miRDB and RNA22 are used to predict 7 target genes for miR-107: AN03, ARMC1, ZHX1, AXIN2, MED26, RNF38 and TMEM47. B. After upregulation of miR-107 expression in Hela and C4-1 cell lines, the expression level of ZHX1 is significantly decreased. C. After down-regulation of miR-107 expression in Hela and C4-1 cell lines, the expression level of ZHX1 is significantly increased. D. miR-107 and ZHX1 3'UTR binding sequences and ZHX1 mutation sequences. E. Luciferase reporter assay. Transfection with wild-type ZHX1 plasmid can significantly reduce the fluorescence intensity, while the fluorescence intensity after transfection with mutant-type ZHX1 plasmid has no significant change.

C4-1 cell line $(P<0.001$, Fig. 4C). The expression levels of ZHX1, DLG1-AS1, and miR-107 were detected in 112 patients with cervical cancer by qRT-PCR. Spearman's correlation test showed that the expression of ZHX1 was positively correlated with that of DLG1-AS1 $(\mathrm{r}=$ $0.569, P<0.001$, Fig. 6A), but negatively correlated with miR-107 expression $(\mathrm{r}=-0.454, P<$ 0.001, Fig. 6B). Therefore, DLG1-AS1, miR-107, and ZHX1 could form a ceRNA network, and DLG1-AS1 could regulate the expression of ZHX1 through competitive binding to miR-107.

Regulation of DLG1-AS1 expression during the proliferation of cervical cancer cells depends on $\mathrm{miR}-107$

To rescue cell proliferation, the HeLa cell line, with relatively high DLG1-AS1 expression, was transfected as follows: Control si+miR-NC, DLG1-AS1 si1+miR-NC, Control si+miR-107 inhibitor, and DLG1-AS1 si1+miR-107 inhibitor. The results showed that down-regulating of both DIG1-AS1 and miR-107 had no significant effect on the proliferation of cervical cancer cells with only down-regulating miR-107 $(P>0.05$, Fig. 7A). The C4- 1 cell line, with low DLG1-AS1 expression, was transfected as follows: Control+miR-NC, DLG1-AS1+miRNC, Control+miR-107 mimic, and DLG1-AS1+miR-107 mimic. The results showed that upregulating of both DIG1-AS1 and miR-107 had no significant effect on the proliferation of cervical cancer cells with only overexpression miR-107 ( $P>0.05$, Fig. 7B), confirming that the regulation of DLG1-AS1 expression during cervical cancer cell proliferation depends on miR-107. 


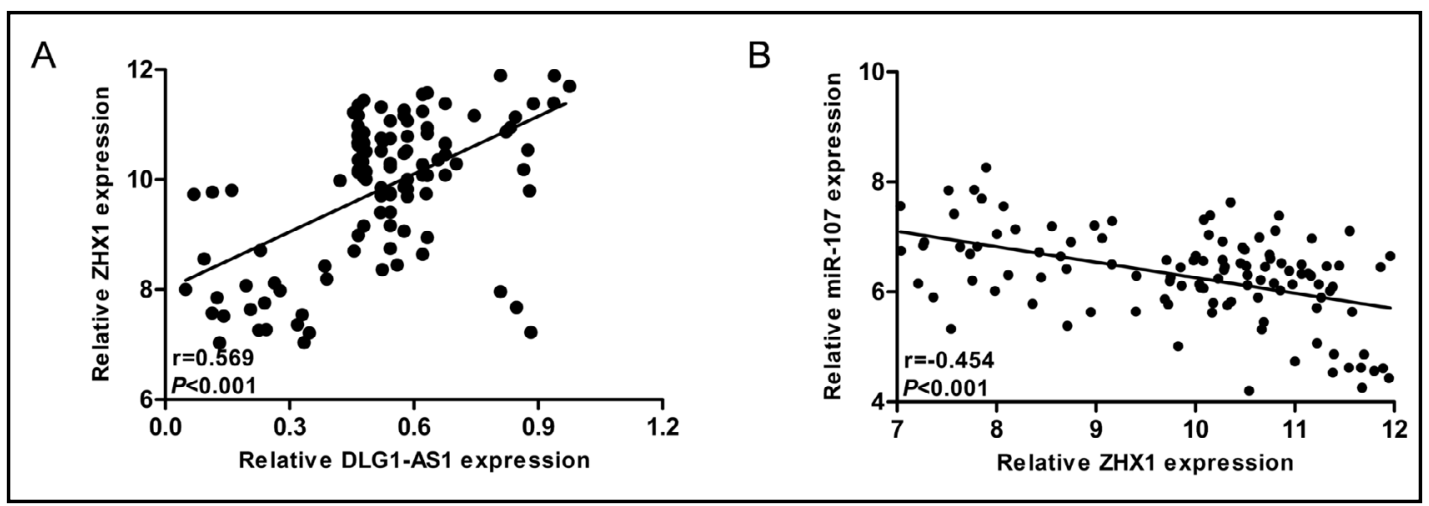

Fig. 6. Correlations of ZHX1 expression with DLG1-AS1 and miR-107 in cervical cancer tissue. A. Correlation between ZHX1 expression and DLG1-AS1 in cervical cancer tissue. The expression level of ZHX1 is positively correlated with DLG1-AS1 ( $\mathrm{r}=0.569, \mathrm{P}<0.001)$. B. Correlation between ZHX1 expression and miR-107 in cervical cancer tissue. The expression level of ZHX1 is negatively correlated with miR-107 ( $r=-0.454$, $\mathrm{P}<0.001)$.

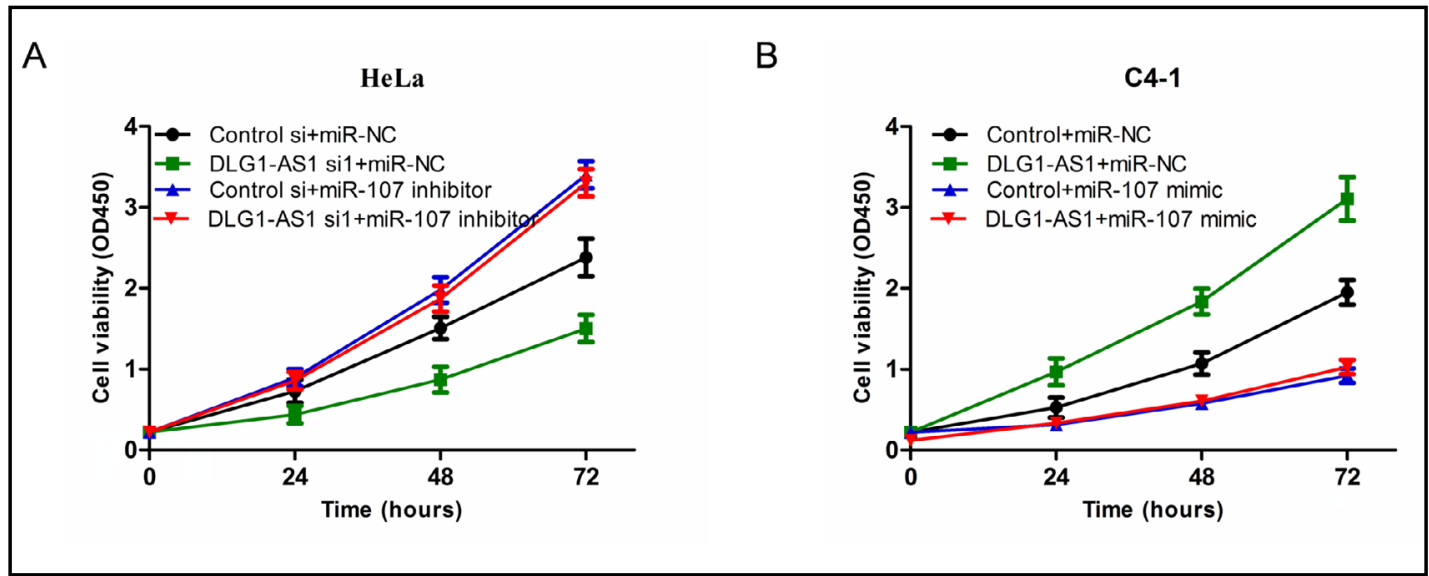

Fig. 7. Regulation of DLG1-AS1 on proliferation of cervical cancer cells depends on miR-107. A. Hela cell lines are transfected as follows: Control si+miR-NC, DLG1-AS1 si1+miR-NC, Control si+miR-107 inhibitor, and DLG1-AS1 si1+miR-107 inhibitor. After down-regulation of DLG1-AS1 expression in the cell line with down-regulated miR-107 expression, the cell proliferation has no significant change $(\mathrm{P}>0.05)$. B. C4-1 cell lines are transfected as follows: Control+miR-NC, DLGl-ASl+miR-NC, Control+miR-107 mimic, and DLGlASl+miR-107 mimic. After up-regulation of DLG1-AS1 expression in the cell line with up-regulated miR-107 expression, the cell proliferation has no significant change $(\mathrm{P}>0.05)$.

\section{Discussion}

The occurrence and development of cervical cancer is a multi-factor and multi-stage process, affected by many oncogenes, tumor suppressor genes, and metastasis-related genes [16]. The expression of these genes is not only regulated at the transcriptional level but also at the post-transcriptional level. Recent studies have revealed that lncRNAs are involved in the occurrence and development of various tumors, including cervical cancer [17-20], attracting increasing attention from researchers. In the present study, IncRNA chip analysis was performed using 10 pairs of cervical cancer and para-carcinoma tissue samples, and 20 lncRNAs with significant differences in their expression were further verified. The results showed that DLG1-AS1 was the most significantly up-regulated lncRNA in cervical cancer tissues and further confirmed that DLG1-AS1 expression was significantly higher in cervical cancer tissues than in para-carcinoma tissues, which was correlated with the prognosis 
of patients with cervical cancer. The OS of patients with high DLG1-AS1 expression was significantly inferior to that of patients with low DLG1-AS1 expression. Therefore, DLG1-AS1 may be a poor prognostic factor for patients with cervical cancer. In cell-based experiments, DLG1-AS1 expression affected the proliferation of cervical cancer cells. After the downregulation of DLG1-AS1 in cervical cancer cells with high DLG1-AS1 expression, their proliferation was reduced, while the up-regulation of DLG1-AS1 in cervical cancer cells with low DLG1-AS1 expression promoted their proliferation, indicating that DLG1-AS1 may lead to tumor progression and poor prognosis by promoting the proliferation of cervical cancer cells.

The mechanism by which DLG1-AS1 as a ceRNA regulates the expression of tumorassociated genes has not been reported. In the present study, bioinformatics databases were used to predict miRNAs that bind to DLG1-AS1. The level of DLG1-AS1 expression was changed in cervical cancer cells to observe quantitative changes in the candidate miRNAs. The results confirmed that miR-107 might be regulated by DLG1-AS1. A dual luciferase reporter assay confirmed that these molecules could bind directly to each other, and the binding site was clearly defined. miR-107 has only one mature sequence that is expressed in many tissues, organs, and gliomas, as well as cervical and prostate cancers, and is involved in the development of tissues and organs as well as the biological functions of various cells. Moreover, this molecule also plays an important regulatory role in the occurrence, migration, and invasion of malignant tumors, showing potential as a new target for the diagnosis and treatment of various tumors. Only a few studies have examined the specific mechanism of miRNA-107 in tumors. Recent studies have shown that miRNA-107 can induce cell cycle arrest in gastric cancer, head-neck squamous cell carcinoma, glioma, and breast cancer by regulating the expression of cell cycle-related target genes, thus inhibiting cell proliferation. In the present study, the correlation between the expression level of miR-107 in cervical cancer tissues and the prognosis of patients was analyzed, and the results confirmed that miR-107 was a positive prognostic factor for cervical cancer. The expression level of miR-107 in cervical cancer tissues was negatively correlated with DLG1-AS1 expression, suggesting that there may be a correlation between these molecules.

It was further determined that ZHX1 was a target gene regulated by miR-107 through database screening, experiments, and dual luciferase reporter assays. ZHX1 belongs to the ZHX protein family, which also includes ZHX2 and ZHX3, and these proteins are expressed widely in human tissues. ZHX1 binds to ZHX2 or ZHX3 to form a heterodimer or binds to itself to form a homodimer and then transfers from the cytoplasm to the nucleus to regulate transcription and participate in important pathophysiological processes [21, 22]. ZHX1 can promote malignant tumor formation. For example, IL-2 can promote the expression of ZHX1 and the proliferation of CTLL-2 cells [23]. In breast cancer, ZHX1 overexpression is associated with cancer cell invasion [24, 25]. In addition, ZHX1 can inhibit B cell differentiation, which is associated with the pathogenesis of Hodgkin's lymphoma [26]. In the present study, the expression level of ZHX1 in cervical cancer tissues was positively correlated with DLG1-AS1 expression, but negatively correlated with miR-107, suggesting that DLG1-AS1, miR-107, and ZHX1 may form a ceRNA network. To confirm that DLG1-AS1 regulates the expression of ZHX1 by competitively binding to miR-107, a cell proliferation rescue assay was performed. After the down-regulation of DLG1-AS1 expression in the HeLa cell line with high DLG1AS1 expression, cell proliferation was reduced, while after the down-regulation of miR-107 expression, cell proliferation was increased. There was no difference in cell proliferation after the simultaneous down-regulation of DLG1-AS1 and miR-107 expression compared with that after the down-regulation of miR-107 alone. Similarly, after the up-regulation of DLG1AS1 in the C4-1 cell line with low DLG1-AS1 expression, cell proliferation was increased, while after the up-regulation of miR-107 expression, cell proliferation was decreased. There was no difference in cell proliferation after the simultaneous up-regulation of DLG1-AS1 and miR-107 compared with that after the up-regulation of miR-107 alone. The above results indicate that the regulation of DLG1-AS1 during the proliferation of cervical cancer cells depends on miR-107.

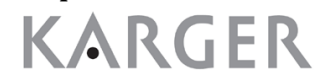




\section{Cellular Physiology Cell Physiol Biochem 2018;49:1792-1803 \begin{tabular}{ll|l} 
and Biochemistry Published online: 20 September, 2018 & $\begin{array}{l}\text { (c) } 2018 \text { The Author(s). Published by S. Karger AG, Basel } \\
\text { www.karger.com/cpb }\end{array}$ \\
\hline
\end{tabular}}

Rui et al.: Effect of IncRNA DLG1-AS1 in Cervical Cancer

\section{Conclusion}

In conclusion, DLG1-AS1/miR-107/ZHX1 can form a ceRNA network, and DLG1-AS1 removes the inhibition of miR-107 on the expression of its target gene ZHX1 by competitively binding to miR-107, thus promoting the proliferation of cervical cancer cells and resulting in tumor progression and the poor prognosis of patients. CeRNAs form a complex RNA regulatory network in cells. One ceRNA can act on several target genes, and the same gene can be regulated by different ceRNAs. Studies on the ceRNA regulatory network will provide a new understanding of the diagnosis, treatment, and prognosis of tumors, and interfering in the regulation between ceRNAs and miRNAs can facilitate the development of new cancer treatment programs. Currently, the exploration of specific regulatory mechanisms of ceRNA networks is still in the preliminary stage. Increasingly more in-depth studies are needed to understand the structure and function of ceRNAs and to clarify the role of ceRNA regulatory networks in the occurrence and development of cervical cancer, thus providing new molecular targets for the diagnosis and treatment of tumors.

\section{Disclosure Statement}

The authors declare that no conflicts of interest exist.

\section{References}

1 Torre LA, Bray F, Siegel RL, Ferlay J, Lortet-Tieulent J, Jemal A: Global cancer statistics, 2012. CA Cancer J Clin 2015;65:87-108.

-2 Chen W, Zheng R, Baade PD, Zhang S, Zeng H, Bray F, Jemal A, Yu XQ, He J: Cancer statistics in China, 2015. CA Cancer J Clin 2016;66:115-132.

-3 Campos-Viguri GE, Jimenez-Wences H, Peralta-Zaragoza O, Torres-Altamirano G, Soto-Flores DG, Hernandez-Sotelo D, Alarcon-Romero Ldel C, Jimenez-Lopez MA, Illades-Aguiar B, Fernandez-Tilapa G: miR$23 \mathrm{~b}$ as a potential tumor suppressor and its regulation by DNA methylation in cervical cancer. Infect Agent Cancer 2015;10:42.

4 Peralta-Zaragoza O, Deas J, Meneses-Acosta A, De la O-Gómez F, Fernandez-Tilapa G, Gomez-Ceron C, Benitez-Boijseauneau O, Burguete-Garcia A, Torres-Poveda K, Bermudez-Morales VH, Madrid-Marina V, Rodriguez-Dorantes M, Hidalgo-Miranda A, Perez-Plasencia C: Relevance of miR-21 in regulation of tumor suppressor gene PTEN in human cervical cancer cells. BMC Cancer 2016;16:215.

-5 Lewis BP, Burge CB, Bartel DP: Conserved seed pairing, often flanked by adenosines, indicates that thousands of human genes are microRNA targets. Cell 2005;120:15-20.

6 Krek A, Grun D, Poy MN, Wolf R, Rosenberg L, Epstein EJ, MacMenamin P, da Piedade I, Gunsalus KC, Stoffel M, Rajewsky N: Combinatorial microRNA target predictions. Nat Genet 2005;37:495-500.

-7 Shao Y, Zhang D, Li X, Yang J, Chen L, Ning Z, Xu Y, Deng G, Tao M, Zhu Y, Jiang J: MicroRNA-203 Increases Cell Radiosensitivity via Directly Targeting Bmi-1 in Hepatocellular Carcinoma. Mol Pharm 2018;15:3205-3215.

$>8$ Wei WF, Zhou CF, Wu XG, He LN, Wu LF, Chen XJ, Yan RM, Zhong M, Yu YH, Liang L, Wang W: MicroRNA221-3p, a TWIST2 target, promotes cervical cancer metastasis by directly targeting THBS2. Cell Death Dis 2017;8:3220.

9 Sannigrahi MK, Sharma R, Singh V, Panda NK, Rattan V, Khullar M: Role of Host miRNA Hsa-miR-139-3p in HPV-16-Induced Carcinomas. Clin Cancer Res 2017;23:3884-3895.

10 Li H, Sheng Y, Zhang Y, Gao N, Deng X, Sheng X: MicroRNA-138 is a potential biomarker and tumor suppressor in human cervical carcinoma by reversely correlated with TCF3 gene. Gynecol Oncol 2017;145:569-576.

11 Huarte M, Rinn JL: Large non-coding RNAs: missing links in cancer? Hum Mol Genet 2010;19:R152-161.

12 Yu F, Chen B, Dong P, Zheng J: HOTAIR Epigenetically Modulates PTEN Expression via MicroRNA-29b: A Novel Mechanism in Regulation of Liver Fibrosis. Mol Ther 2017;25:205-217. 


\section{Cellular Physiology Cell Physiol Biochem 2018;49:1792-1803

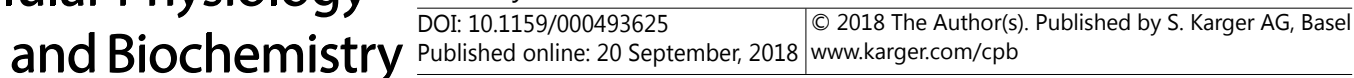 \\ Rui et al.: Effect of IncRNA DLG1-AS1 in Cervical Cancer}

13 Yue B, Cai D, Liu C, Fang C, Yan D: Linc00152 Functions as a Competing Endogenous RNA to Confer Oxaliplatin Resistance and Holds Prognostic Values in Colon Cancer. Mol Ther 2016;24:2064-2077.

14 Teng H, Wang P, Xue Y, Liu X, Ma J, Cai H, Xi Z, Li Z, Liu Y: Role of HCP5-miR-139-RUNX1 Feedback Loop in Regulating Malignant Behavior of Glioma Cells. Mol Ther 2016;24:1806-1822.

-15 Liu Q Hu X, Zhang X, Dai L, Duan X, Zhou C, Ao Y: The TMSB4 Pseudogene LncRNA Functions as a Competing Endogenous RNA to Promote Cartilage Degradation in Human Osteoarthritis. Mol Ther 2016;24:1726-1733.

16 Chen X, Leung SY, Yuen ST, Chu KM, Ji J, Li R, Chan AS, Law S, Troyanskaya OG, Wong J, So S, Botstein D, Brown PO: Variation in gene expression patterns in human gastric cancers. Mol Biol Cell 2003;14:32083215.

17 Dong J, Wang Q, Li L, Xiao-Jin Z: Upregulation of Long Non-Coding RNA Small Nucleolar RNA Host Gene 12 Contributes to Cell Growth and Invasion in Cervical Cancer by Acting as a Sponge for MiR-424-5p. Cell Physiol Biochem 2018;45:2086-2094.

18 Mao X, Qin X, Li L, Zhou J, Zhou M, Li X, Xu Y, Yuan L, Liu QN, Xing H: A 15-long non-coding RNA signature to improve prognosis prediction of cervical squamous cell carcinoma. Gynecol Oncol 2018;149:181-187.

19 Guo H, Yang S, Li S, Yan M, Li L, Zhang H: LncRNA SNHG20 promotes cell proliferation and invasion via miR140-5p-ADAM10 axis in cervical cancer. Biomed Pharmacother 2018;102:749-757.

20 Li H, Jia Y, Cheng J, Liu G, Song F: LncRNA NCK1-AS1 promotes proliferation and induces cell cycle progression by crosstalk NCK1-AS1/miR-6857/CDK1 pathway. Cell Death Dis 2018;9:198.

21 Yamada K, Printz RL, Osawa H, Granner DK: Human ZHX1: cloning, chromosomal location, and interaction with transcription factor NF-Y. Biochem Biophys Res Commun 1999;261:614-621.

22 Yamada K, Kawata H, Shou Z, Hirano S, Mizutani T, Yazawa T, Sekiguchi T, Yoshino M, Kajitani T, Miyamoto $\mathrm{K}$ : Analysis of zinc-fingers and homeoboxes (ZHX)-1-interacting proteins: molecular cloning and characterization of a member of the ZHX family, ZHX3. Biochem J 2003;373:167-178.

-23 Shou Z, Yamada K, Kawata H, Yokoyama O, Miyamoto K: A mechanism of induction of the mouse zincfingers and homeoboxes 1 (ZHX1) gene expression by interleukin-2. Biochem Biophys Res Commun 2004;314:885-890.

24 Firlej V, Ladam F, Brysbaert G, Dumont P, Fuks F, de Launoit Y, Benecke A, Chotteau-Lelievre A: Reduced tumorigenesis in mouse mammary cancer cells following inhibition of Pea3- or Erm-dependent transcription. J Cell Sci 2008;121:3393-3402.

25 Chen S, Yu X, Lei Q, Ma L, Guo D: The SUMOylation of zinc-fingers and homeoboxes 1 (ZHX1) by Ubc9 regulates its stability and transcriptional repression activity. J Cell Biochem 2013;114:2323-2333.

26 Nagel S, Ehrentraut S, Meyer C, Kaufmann M, Drexler HG, MacLeod RA: Aberrantly Expressed OTX Homeobox Genes Deregulate B-Cell Differentiation in Hodgkin Lymphoma. PLoS One 2015;10:e0138416. 